\title{
Acral Persistent Papular Mucinosis associated with Multiple Myeloma - Unknown Presentation
}

\author{
Mushtaq Ahmad, M.D., Farhana Siraj, M.D., Mubashir Ahmad, M.D., Shabir Ahmad, M.D. \\ Department of Medicine, Sher-i-Kashmir Institute of Medical Sciences, Srinagar
}

\section{A B S T R A C T}

The cutaneous mucinosis are a heterogenous group of disorders in which mucin accumulates in the skin. A 66 years old man presented with asymptomatic flesh coloured papules on the extremities without any truncal involvement of 3 months duration. Histopathological examination of a lesional biopsy revealed unremarkable epidermis with dermis showing pallor and loosening of collagen bundles in mucin like deposition suggestive of cutaneous mucinosis. Patient was put on steroids and hydroxycholoroquinine. Three months later he presented with encephalopathy. Investigation revealed hypercalcaemia, reversal of albumin globulin ratio, azotemia. X-ray skull revealed lytic lesions. Bone marrow aspiration and bone marrow biopsy was suggestive of multiple myeloma. This is an unknown presentation, as localized cutaneous mucinosis is not associated with systemic disease. Patient was started on chemotherapy for multiple myeloma. JMS 2012;15(1):74-75.

KeyWords: Mucinosis, hydroxycholoroquinine, multiple myeloma, chemotherapy

A 66 year old man presented 3 months back to dermatologist with the complaints of asymptomatic flesh colored lesions on forearms, hands, lower limbs below knees and feet. on dermatological examination patient was found to have papulo nodular lesions on the extremeties without any lesion on the truncal region. Baseline investigation including complete blood count, liver function tests, Kidney function tests were normal. Antinuclear antibodies were negative. Skin biopsy of the lesion was showing epidermis unremarkable with dermal pallor and loosening of collagen bundles in mucin like deposition suggestive of cutaneous mucinosis[Figure]. Patient was put on oral steroid and hydroxychloroquine. On followup patient did not had

\section{Correspondence:}

Dr. Mushtaq Ahmad

Assistant Prof., Department of Medicine

Sher-i-Kashmir Institute of Medical Sciences, Srinagar

E-Mail:drmushtaq_01@yahoo.co.in much resolution of these skin lesions and presently patient presented with complaints of altered sensorium, recurrent vomiting, anorexia, and constipation .General physical examination revealed that patient was dehydrated there were papulonodular lesions over the extremities. The distribution of the lesions was symmetrical, involving both upper and lower limbs. Upper limb involvement was from below elbows involving forearms and hands.Lower limb involvement was below knees including feet. Chest, cardiovascular and perabdomen examination was normal. Nervous system examination revealed that patient was in grade II encephalopathy without any signs of meningeal irritation or focal neurological deficit. Complete blood count was normal. liver function tests were showing hypergammaglobinnemia with reversal of albumin globulin ratio. Kidney function tests revealed azotemia and serum calcium levels were repeatedly high. Skeletal survey revealed punched out lytic lesions in skull x-ray. Non contrast computed tomography was normal. Serum electrolytes 


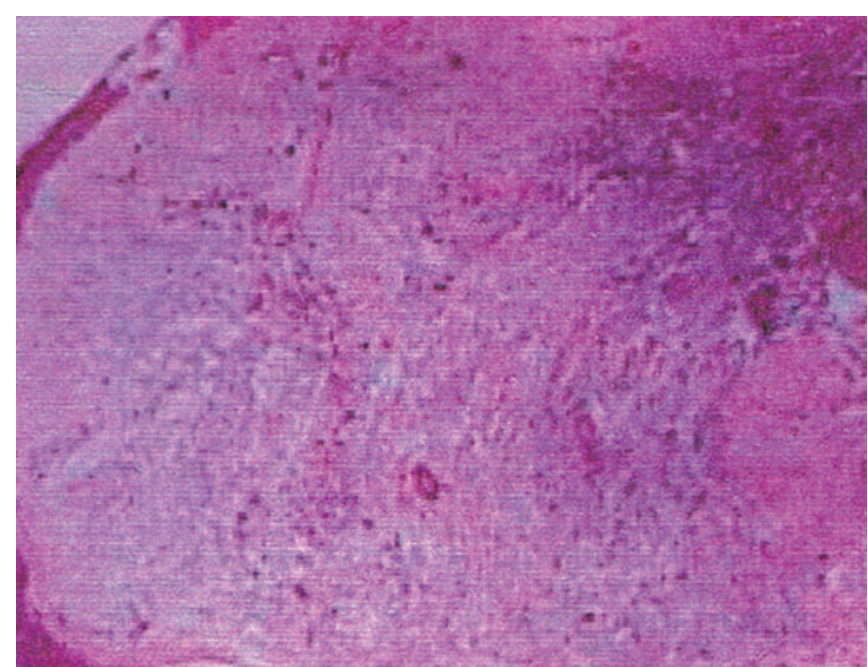

FIGURE. Biopsy of shin showing epidermis unremarkable with dermal pallor and loosening of collagen bundles in mucin like deposition suggestive of cutaneous mucinosis

revealed hypernatremia. Ultrasound abdomen for hepatobiliary system and kidneys was normal. Bone marrow biopsy was suggestive of Multiple myeloma.

\section{Discussion}

Papular mucinosis was first described in 1906[1]. It is classified into three types: Generalised papular, localized and atypical form. localized form is further divided into various forms. All forms require mucin deposition and absence of thyroid disease. Diagnosis of generalised form requires febroblast proliferation and fibrosis on histopathologic examination and an associated monoclonal gammopathy. Diagnosis of localized form requires the absence of a monoclonal gammopathy. The atypical subtypes include scleromyxedema without monoclonal gammopathy or localized form with monoclonal gammopathy or systemic symptoms other than HIV infection, localized mixed features of diffrent subtypes and other not well difined cases[2,3]. All forms of lichen myxedematosus present with firim, dome - shaped waxy papules, nodules, or plaques. Rare cases of localized LM are associated with monoclonal gammopathy. They include a patient with acral persistant popular mucinosis and IgA paraproteinemia and another cases of discrete papular mucinosis with an abnormal serum protien $[4,5]$. case of acral persistent papular mucinosis with multiple myeloma was not found in literature to our best effort to find such association. The case discussed presented with symtomatic hypercalcaemia and had encephalopathy secondary to hypernatraemia. Hypercalcaemia and hypernatraemia responded to fluid therapy and was subjected to definitive therapy of Multiple myeloma.

\section{References}

1. Dubreuilh W. Fibromes miliaries folliculaires: sclerodermie consecutive. Arch Dermatol Syplilol 1906; $7 ; 569$.

2. Rengioletti F, Reboro A. updated classification of papular mucinosis, lichen myxedematosus, and scleromyxedema.JAm Acad Dermatol 2001;444;273.

3. Rongioletti F. Lichen myxedematosus (papular mucinosis): new concepts and perspectives for an old disease. Semincutan Med Surg 2006;25:100.

4. Harde RA, Hunter JA, Urbaniak S, Habeshaw JA. Br J Dermatol 1979;100:727-30.

5. Borradori L, Aractingi S, Blanc F, Verbolao, Dubertret L. Dermatology 1992;185:134-6. 